\title{
Itô calculus and quantisation of Lie bialgebras
}

\author{
R.L. Hudson* \\ Nottingham Trent University, School of Computing and Mathematics, Burton Street, Nottingham, NG1 4BU, UK
}

Received 17 February 2004; accepted 24 September 2004

Available online 29 March 2005

\begin{abstract}
Motivated by classical and quantum stochastic flows, particularly the distinction between inner and outer flows in the quantum case, we develop analogous notions which live in the Itô Hopf algebra, which is got by replacing the shuffle product in a shuffle Hopf algebra by a noncommutative multiplication which abstracts the product rule for iterated quantum stochastic integrals. Inner flows got by conjugation by ordered double product integrals $\overleftrightarrow{\prod}(1+\mathrm{d} r[h])$ are used to quantise Lie bialgebras. (c) 2005 Elsevier SAS. All rights reserved.

\section{Résumé}

Nous sommes motivés par les flots classiques et quantiques, notamment par la distinction entre les flots intérieurs et les flots extérieurs dans le cas quantique. Nous développons les notions analogues dans l'algèbre de Hopf-Itô, qui est obtenue en remplaçant le produit dans l'algèbre de battage par un produit non commutatif qui imite la multiplication des intégrales stochastiques quantiques itérées. Les flots intérieurs obtenus par conjugaison par les intégrales multiplicatives doubles ordonnées $\vec{\prod}(1+\mathrm{d} r[h])$ sont utilisés pour la quantification des bialgébres de Lie.
\end{abstract}

(c) 2005 Elsevier SAS. All rights reserved.

MSC: $81 \mathrm{~S} 25 ; 17 \mathrm{~B} 62$

\section{Introduction}

P.A. Meyer [10] emphasized that an algebraic viewpoint on stochastic flows is necessary to obtain a satisfactory quantum generalisation. This follows the viewpoint of Accardi, Frigerio and Lewis [1] that a quantum random variable is a homomorphism of associative algebras. Thus, for a stochastic flow, instead of a description by a stochastic differential equation which directly describes the evolution of a time dependent random point $X_{t}$ starting at a generic point of a state space, one considers a suitable algebra $\mathcal{A}$ of functions on the state space and describes

\footnotetext{
* Tel.: +44 115 8484314; fax: +44 1159514933 .

E-mail address: robin.hudson@ntu.ac.uk (R.L. Hudson).
} 
the flow by means of the time dependent family of homomorphisms $J_{t}$, where $J_{t}(f)=f\left(X_{t}^{*}\right)$, which take $\mathcal{A}$ into a larger algebra $\tilde{\mathcal{A}}$ of functions on the Cartesian product of the state space with the underlying probability space $\Omega$. Corresponding to the stochastic differential equation for $X_{t}$, one obtains a stochastic differential equation for $J$ which, for example in the case of a flow driven by an $n$-dimensional Brownian motion $\left(B_{1}, B_{2}, \ldots, B_{n}\right)$, takes the form

$$
\mathrm{d} J_{t}=\sum_{j=1}^{n} J \circ \beta^{(j)} \mathrm{d} B_{j}+J \circ \tau \mathrm{d} t, \quad J_{0}(f)=f \otimes \mathrm{id}_{\Omega} .
$$

Here $\beta^{(1)}, \beta^{(2)}, \ldots, \beta^{(n)}$ and $\tau$ are linear maps from $\mathcal{A}$ to itself called structure maps, which, corresponding to the multiplicativity of $J_{t}$ and the Itô product rules $\mathrm{d} B_{j} \mathrm{~d} B_{k}=\delta_{j, k} \mathrm{~d} t$, must satisfy the socalled structure relations

$$
\beta^{(j)}(f g)=\beta^{(j)}(f) g+f \beta^{(j)}(g), \quad \tau(f g)=\tau(f) g+f \tau(g)+\sum_{j=1}^{n} \beta^{(j)}(f) \beta^{(j)}(g) .
$$

The quantum generalisation is twofold. First, there is in general no longer any state space so that now $\mathcal{A}$ is a noncommutative algebra, typically the algebra $B(\mathcal{H})$ of all bounded operators on a system Hilbert space $\mathcal{H}$. Secondly the Ito algebra, which in the classical situation above is the complex associative algebra spanned by the Itô differentials $\mathrm{d} B^{(1)}, \mathrm{d} B^{(2)}, \ldots, \mathrm{d} B^{(n)}, \mathrm{d} t$ equipped with the Itô product rules, is replaced by a noncommutative associative algebra $\mathcal{L}$, typically spanned by differentials $\mathrm{d} \Lambda_{\beta}^{\alpha}, \alpha, \beta=0,1,2, \ldots, n$, with the multiplication rule $\mathrm{d} \Lambda_{\beta}^{\alpha} \mathrm{d} \Lambda_{\varepsilon}^{\gamma}=\left(1-\delta_{0}^{\alpha}\right) \delta_{\varepsilon}^{\alpha} \mathrm{d} \Lambda_{\beta}^{\gamma}$. These correspond to operator-valued processes $\Lambda_{\beta}^{\alpha}, \alpha, \beta=0,1,2, \ldots, n$, living in the Fock space $\mathcal{F}$ over the Hilbert space $L^{2}\left(\mathbb{R}^{+} ; \mathbb{C}^{n}\right)$ for which there is a corresponding theory of stochastic integration [5]. The case of flows driven by Brownian motion can be recovered by restriction to the mutually commuting, self-adjoint processes $B_{j}=\Lambda_{0}^{j}+\Lambda_{j}^{0}$ and the time $t=\Lambda_{0}^{0}$.

There is a canonical splitting of Fock space at each time $t \in \mathbb{R}^{+}$into the Hilbert space tensor product $\mathcal{F}=$ $\mathcal{F}_{t} \otimes \mathcal{F}^{t}$ of the Fock spaces with $\mathbb{R}^{+}$replaced by the intervals $[0, t[$ and $[t, \infty[$ respectively, with respect to which each operator $\Lambda_{\beta}^{\alpha}(t)$ is the ampliation to $\mathcal{F}$ by the identity operator in the future sector $\mathcal{F}^{t}$ of an operator in the past sector $\mathcal{F}_{t}$ and similarly each increment $\Lambda_{\beta}^{\alpha}(s)-\Lambda_{\beta}^{\alpha}(t)$ with $s>t$ is the ampliation to $\mathcal{F}$ of an operator in the future sector. Splitting gives a natural notion of adaptedness and allows an interpretation in which all the basic processes $\Lambda_{\beta}^{\alpha}$ have independent increments.

In this context a quantum stochastic flow is described by a family $\left(J_{t}\right)_{t \in \mathbb{R}^{+}}$of $C^{*}$ - or von Neumann algebra homomorphisms from $B(\mathcal{H})$ to $B(\mathcal{H} \otimes \mathcal{F})$ satisfying

$$
\mathrm{d} J=\sum_{\alpha, \beta=0}^{n} J \circ \lambda_{\beta}^{\alpha} \mathrm{d} \Lambda_{\alpha}^{\beta}, \quad J_{0}(x)=x \otimes 1_{B(\mathcal{F})}
$$

where the structure maps $\lambda_{\beta}^{\alpha}: B(\mathcal{H}) \rightarrow B(\mathcal{H})$ satisfy the structure relations

$$
\lambda_{\beta}^{\alpha}(x y)=\lambda_{\beta}^{\alpha}(x) y+x \lambda_{\beta}^{\alpha}(y)+\sum_{j=1}^{n} \lambda_{j}^{\alpha}(x) \lambda_{\beta}^{j}(y) .
$$

More concisely we may write

$$
\mathrm{d} J=J^{1,2} j^{1,3}, \quad J_{0}=\mathrm{id}_{B(\mathcal{H})} \otimes 1_{B(F)},
$$

where the superscripts are place notation indicating in which factors of the triple tensor product space $B(\mathcal{H} \otimes \mathcal{F}) \otimes$ $\mathcal{L}=B(\mathcal{H}) \otimes B(\mathcal{F}) \otimes \mathcal{L}$ the relevant map lives, and the generator $j$ of the flow is the map $\sum_{\alpha, \beta=0}^{n} \lambda_{\beta}^{\alpha}(\cdot) \otimes \mathrm{d} \Lambda_{\alpha}^{\beta}$ from $B(\mathcal{H})$ to $B(\mathcal{H}) \otimes \mathcal{L}$, and satisfies the structure relation

$$
j(x y)=j(x) y+x j(y)+j(x) j(y) .
$$


A flow $J$ is called inner if its generator $j$ takes the form

$$
j(x)=u x+x v+u x v
$$

where $u$ and $v$ are elements of $B(\mathcal{H}) \otimes \mathcal{L}$ which are mutually quasiinverse in the sense that

$$
u+v+u v=v+u+v u=0 .
$$

Correspondingly $J$ takes the form

$$
J(x)=U\left(x \otimes 1_{B(F)}\right) V
$$

where $U$, and $V$ are the mutually inverse operator valued processes solving the stochastic differential equations

$$
\mathrm{d} U=U^{1,2} u^{1,3}, \quad U_{0}=1_{B(H)} \otimes 1_{B(F)}, \quad \mathrm{d} V=v^{1,3} V^{1,2}, \quad V_{0}=1_{B(H)} \otimes 1_{B(F)} .
$$

Note that the concept of an inner flow has no classical counterpart. For comparison with what follows, it is useful to introduce the product integral notations

$$
J=\prod(\mathrm{id}+\mathrm{d} j), \quad U=\vec{\prod}(1+\mathrm{d} u), \quad V=\overleftarrow{\prod}(1+\mathrm{d} v)
$$

for the flow $J$ generated by $j$ and the operator-valued processes solving (1).

The purpose of this paper can now be explained. We first equip the space of tensors $\mathcal{T}(\mathcal{L})$ over an arbitrary finite-dimensional associative algebra $\mathcal{L}$ with operations which make it a Hopf algebra, called the Itô Hopf algebra, which is a noncommutative generalisation of the Hopf algebra based on the shuffle product in $\mathcal{T}(\mathcal{L})$ used in [2], to which it reduces in the case when the multiplication in $\mathcal{L}$ is the trivial one for which all products vanish. When $\mathcal{L}$ is either the quantum or classical algebras of Itô differentials spanned by $\mathrm{d} B^{(1)}, \mathrm{d} B^{(2)}, \ldots, \mathrm{d} B^{(n)}, \mathrm{d} t$ or by $\mathrm{d} \Lambda_{\beta}^{\alpha}, \alpha, \beta=0,1,2, \ldots, n$, the multiplication in the Itô Hopf algebra corresponds to the multiplication formula for iterated stochastic integrals. The coproduct is similarly related to the property of independent increments in these cases. It is convenient to introduce right and left differential maps $\vec{d}$ and $\overleftarrow{d}$ in $\mathcal{T}(\mathcal{L})$ corresponding to both the usual forward derivative and its backward counterpart in the stochastic case. Then we can define simple product integrals and product flows

$$
\prod(1+\mathrm{d} L[h]), \quad \prod(\mathrm{id}+\mathrm{d} j[h]) .
$$

The first of these is an element of the space $\mathcal{T}(\mathcal{L}) \llbracket h \rrbracket$ of formal power series with coefficients in $\mathcal{T}(\mathcal{L})$ which is generated by an element $L[h]$ of $h \mathcal{L} \llbracket h \rrbracket$. The second is a linear map from $\mathcal{T}(\mathcal{L})$ to $\mathcal{T}(\mathcal{L}) \llbracket h \rrbracket$ and is generated by a linear map $j[h]$ from $\mathcal{L}$ to $\mathcal{L} \llbracket h \rrbracket$. Both are defined as solutions of differential equations, but they can also be characterised by algebraic properties. The inverse of $\prod(1+\mathrm{d} L[h])$ is $\prod\left(1+\mathrm{d} L^{\prime}[h]\right)$ where $L^{\prime}[h]$ is the quasiinverse of $L[h]$ in $h \mathcal{L} \llbracket h \rrbracket$; conjugation by $\prod(1+\mathrm{d} r L[h])$ is then an inner product flow which is multiplicative.

Of more use for applications to quantisation is the theory of double product integrals

$$
\vec{\prod}(1+\mathrm{d} r[h]), \quad \overleftrightarrow{\prod}\left(1+\mathrm{d} r^{\prime}[h]\right)
$$

which are elements of $(\mathcal{T}(\mathcal{L}) \otimes T(L)) \llbracket h \rrbracket$ with generators $r[h]$ and $r^{\prime}[h]$ in $h(\mathcal{L} \otimes \mathcal{L}) \llbracket h \rrbracket$. Unlike simple product integrals, their definitions incorporate directional senses. From the point of view of stochastic calculus in Fock space, regarded through splitting as a continuous tensor product [11], $\vec{\prod}(1+\mathrm{d} r[h])$ may be regarded as a continuous analog, living in the double Fock space $\mathcal{F} \otimes \mathcal{F}$, of a discrete double product of the form

$$
\prod_{(j, k) \in \mathbb{N}_{m} \times \mathbb{N}_{n}} x^{j ; k}=\prod_{j \in \mathbb{N}_{m}}^{\rightarrow}\left\{\prod_{k \in \mathbb{N}_{n}} x^{j ; k}\right\}=\prod_{k \in \mathbb{N}_{n}}^{\overleftarrow{ }}\left\{\prod_{j \in \mathbb{N}_{m}} x^{j ; k}\right\}
$$

in which $x$ is an element of a tensor product algebra $\mathcal{A} \otimes \mathcal{A}$ and the place notation $x^{j ; k}$ indicates that $x$ occupies the tensor product of the $j$-th copy of $\mathcal{A}$ within $\otimes^{m} \mathcal{A}$ and the $k$-th copy of $\mathcal{A}$ within $\otimes^{n} \mathcal{A}$ so that the two iterated 
products (which are indeed equal [9]) are elements of $\left(\otimes^{m} \mathcal{A}\right) \otimes\left(\otimes^{n} \mathcal{A}\right)$. However such a Fock space interpretation is inhibited by severe divergence difficulties when the formal parameter $h$ is replaced by a complex variable [9].

Though once again they can be defined as solutions of stochastic differential equations, forward-backward double product integrals $R[h]=\vec{\prod}(1+\mathrm{d} r[h])$ can be characterised algebraically [7] by the socalled quasitriangularity relations which form the starting point of Enriquez's approach to the quantisation problem for Lie bialgebras [2]. The second stage of his approach is to require that $R[h]$ satisfy the quantum Yang-Baxter equation and there is a necessary and sufficient condition on the generator $r[h]$ for this [8]. Using the fact that $\vec{\prod}(1+\mathrm{d} r[h])$ and $\overleftrightarrow{\prod}\left(1+\mathrm{d} r^{\prime}[h]\right)$ are mutual inverses if $r[h]$ and $r^{\prime}[h]$ are mutually quasiinverse one may then construct a deformation coproduct by composing the coproduct in $\mathcal{T}(\mathcal{L})$ with conjugation by the invertible element $\vec{\prod}(1+\mathrm{d} r[h])$ of $(\mathcal{T}(\mathcal{L}) \otimes \mathcal{T}(\mathcal{L})) \llbracket h \rrbracket$. In this way we obtain a simple and general procedure for quantising Lie bialgebras of quasitriangular type in which the Lie bracket is got by taking commutators in an associative algebra.

It is natural to regard the map got by conjugation by $\vec{\prod}(1+\mathrm{d} r[h])$ as an inner double flow and to seek a more general notion of double flow with the expectation that these will similarly quantise Lie bialgebras of a more general type.

We use the following notations. $\mathbb{N}$ denotes the set of natural numbers and, for $l<m$ belonging to $\mathbb{N}, \mathbb{N}_{m}^{l}$ and $\mathbb{N}_{m}$ denote its subsets $\{l+1, l+2, \ldots, m\}$ and $\{1,2, \ldots, m\}$.

\section{The Itô Hopf algebra}

Let $\mathcal{L}$ be a not necessarily unital complex associative algebra. We may think of $\mathcal{L}$ as the Itô algebra of differentials in a classical or quantum stochastic calculus.

Let $\mathcal{T}(\mathcal{L})=\bigoplus_{n=0}^{\infty}\left(\bigotimes^{n} \mathcal{L}\right)$ denote the space of all tensors over $\mathcal{L}$. Thus elements of $\mathcal{L}$ are sequences $\alpha=$ $\left(\alpha_{0}, \alpha_{1}, \alpha_{2}, \ldots\right)$ where each $\alpha_{n}$ is a homogeneous tensor of rank $n$, in which only finitely many $\alpha_{n}$ are nonzero. Generalising the well known shuffle product, which is obtained when $\mathcal{L}$ has the trivial multiplication where all products vanish, we equip $\mathcal{T}(L)$ with the product defined by bilinear extension of the rule

$$
\left(L_{1} \otimes L_{2} \otimes \cdots \otimes L_{m}\right)\left(L_{m+1} \otimes L_{m+2} \otimes \cdots \otimes L_{m+n}\right)=\sum_{P \in \mathcal{P}_{m, n}}\left(L_{P_{1}} \otimes L_{P_{2}} \otimes \cdots \otimes L_{P_{k}}\right)
$$

where $\mathcal{P}_{m, n}$ is the set of Itô shuffles (or sticky shuffles, in which a card from the first pack may stick to a card from the second), that is, ordered partitions $P=\left(P_{1}, P_{2}, \ldots, P_{k}\right)$ of $\{1,2, \ldots, m+n\}$ into subsets $P_{j}$ which are either singletons $\{s\}$, in which case $L_{P_{j}}=L_{s}$, or pairs $(s, t)$ with $s \in\{1,2, \ldots, m\}$ and $t \in\{m+1, m+2, \ldots, m+n\}$, in which case $L_{P_{j}}=L_{s} L_{t}$, and in which the subsets $\{1,2, \ldots, m\}$ and $\{m+1, m+2, \ldots, m+n\}$ retain their natural relative orders in the ordered set got by deleting internal brackets in $\left(P_{1}, P_{2}, \ldots, P_{k}\right)$.

When $\mathcal{L}$ is an Itô algebra, the multiplication (1) can be understood as follows. We fix a finite subinterval $[a, b[$ of the real half-line $\mathbb{R}^{+}$, and map each homogeneous product tensor to a corresponding iterated stochastic integral

$$
I_{a}^{b}\left(L_{1} \otimes L_{2} \otimes \cdots \otimes L_{m}\right)=\int_{a<t_{1}<t_{2}<\cdots<t_{m}<b} \mathrm{~d} L_{1}\left(t_{1}\right) \mathrm{d} L_{2}\left(t_{2}\right) \cdots \mathrm{d} L_{m}\left(t_{m}\right) .
$$

Then (1) gives the multiplication rule for such integrals:

$$
I_{a}^{b}\left(L_{1} \otimes L_{2} \otimes \cdots \otimes L_{m}\right) I_{a}^{b}\left(L_{m+1} \otimes L_{m+2} \otimes \cdots \otimes L_{m+n}\right)=\sum_{P \in \mathcal{P}_{m, n}} I_{a}^{b}\left(L_{P_{1}} \otimes L_{P_{2}} \otimes \cdots \otimes L_{P_{k}}\right) .
$$


Alternatively we may describe the multiplication in $\mathcal{T}(\mathcal{L})$ by $\alpha \beta=\gamma$ where the homogeneous components of the tensor $\gamma$ are given in terms of those of $\alpha$ and $\beta$ by

$$
\gamma_{n}=\sum_{A \cup B=\mathbb{N}_{n}} \alpha_{|A|}^{A} \beta_{|B|}^{B} .
$$

Here and subsequently we use the place notation that, for example, $\alpha_{|A|}^{A}$ indicates that the $|A|$-th rank homogeneous component $\alpha_{|A|} \in \bigotimes^{|A|} \mathcal{L}$ of $\alpha$ is placed in the tensor product of those copies of $\mathcal{L}$ within the $n$-fold tensor product $\bigotimes^{n} \mathcal{L}$ labelled by the elements of $A$. Since $A \cup B=\{1,2, \ldots, n\}$ all copies of $\mathcal{L}$ in $\bigotimes^{n} \mathcal{L}$ are occupied in the combination $\alpha_{|A|}^{A} \beta_{|B|}^{B}$; when $A \cap B \neq \emptyset$ double occupancies of $\mathcal{L}$ are resolved using the multiplication in $\mathcal{L}$.

It is well known that the shuffle product algebra becomes a Hopf algebra when equipped with the coproduct $\Delta$ defined by linear extension of the rule

$$
\Delta\left(L_{1} \otimes L_{2} \otimes \cdots \otimes L_{m}\right)=\sum_{j=0}^{m}\left(L_{1} \otimes L_{2} \otimes \cdots \otimes L_{j}\right) \otimes\left(L_{j+1} \otimes L_{j+2} \otimes \cdots \otimes L_{m}\right) .
$$

The counit $\varepsilon$ is given by

$$
\varepsilon\left(\alpha_{0}, \alpha_{1}, \alpha_{2}, \ldots\right)=\alpha_{0}
$$

and the antipode by linear extension of the map

$$
L_{1} \otimes L_{2} \otimes \cdots \otimes L_{m} \longmapsto(-1)^{m}\left(L_{m} \otimes L_{m-1} \otimes \cdots \otimes L_{1}\right) .
$$

It can be shown [7] that $\Delta$ remains a coproduct, with the same counit $\varepsilon$, when the shuffle product is replaced by the Itô shuffle product (1), and makes the Itô shuffle algebra $\mathcal{T}(\mathcal{L})$ into a Hopf algebra, the Itô Hopf algebra, when equipped with an antipode $S$ which is a deformation of (5) in the sense that

$$
S\left(L_{1} \otimes L_{2} \otimes \cdots \otimes L_{m}\right)=(-1)^{m}\left(L_{m} \otimes L_{m-1} \otimes \cdots \otimes L_{1}\right)+\text { terms of rank }<m .
$$

When $\mathcal{L}$ is an Itô algebra, the coproduct can be given a simple interpretation in Fock space quantum stochastic calculus as follows. Let $a<b<c \in \mathbb{R}^{+}$. Then, corresponding to the canonical splitting of Fock space at time $b$, we have

$$
I_{a}^{c}=\left(I_{a}^{b} \otimes I_{b}^{c}\right) \Delta
$$

where the ranges of $I_{a}^{b}$ and $I_{b}^{c}$ are identified with their preampliations in the past and future Fock spaces of the splitting at time $b$.

We shall find the following observation useful. Denote by $\Delta^{(n)}, n=1,2, \ldots$, the iterated coproducts mapping $\mathcal{T}(\mathcal{L})$ to $\bigotimes^{n} \mathcal{T}(\mathcal{L})$, defined by $\Delta^{(1)}=\operatorname{id}_{\mathcal{T}(\mathcal{L})}, \Delta^{(2)}=\Delta$, and for $n>2$,

$$
\Delta^{(n)}=\left(\Delta \otimes \operatorname{id}_{\otimes^{n-2} \mathcal{T}(\mathcal{L})}\right) \Delta^{(n-1)} .
$$

Then the $n$-th rank homogeneous component $\alpha_{n} \in \bigotimes^{n} \mathcal{L}$ of an element $\alpha=\left(\alpha_{0}, \alpha_{1}, \alpha_{2}, \ldots\right)$ of $\mathcal{T}(\mathcal{L})$ is equal to the component of joint rank $(1,1, \ldots, 1)$ of $\Delta^{(n)} \alpha$ in

$$
\begin{aligned}
& \bigotimes^{n} \mathcal{T}(\mathcal{L})=\bigoplus_{m_{1}, m_{2}, \ldots, m_{n}=0}^{\infty}\left(\bigotimes_{\substack{(n) . \\
(1,1, \ldots, 1)}}^{m_{1}} \mathcal{L}\right) \otimes\left(\bigotimes^{m_{2}} \mathcal{L}\right) \otimes \cdots \otimes\left(\bigotimes^{m_{n}} \mathcal{L}\right), \\
& \alpha_{n}=\left(\Delta^{(n)} \alpha\right)
\end{aligned}
$$

The subspace $\mathcal{S}(\mathcal{L})$ of $\mathcal{T}(\mathcal{L})$ consisting of symmetric tensors is a sub-Hopf algebra of the Itô Hopf algebra, isomorphic as a Hopf algebra to the universal enveloping algebra $\mathcal{U}$ of the Lie algebra got by equipping $\mathcal{L}$ with the commutator Lie bracket, under the universal extension of the Lie algebra homoorphism $\mathcal{L} \ni L \mapsto(0, L, 0,0, \ldots) \in$ $\mathcal{S}(\mathcal{L})[4]$. 


\section{Calculus}

We define right and left differential maps $\vec{d}$ from $\mathcal{T}(\mathcal{L})$ to $\mathcal{T}(\mathcal{L}) \otimes L$ and $\overleftarrow{d}$ from $\mathcal{T}(\mathcal{L})$ to $\mathcal{L} \otimes \mathcal{T}(\mathcal{L})$ by linear extension of

$$
\begin{aligned}
& \vec{d}\left(L_{1} \otimes L_{2} \otimes \cdots \otimes L_{m}\right)=\left(L_{1} \otimes L_{2} \otimes \cdots \otimes L_{m-1}\right) \otimes L_{m} \\
& \overleftarrow{d}\left(L_{1} \otimes L_{2} \otimes \cdots \otimes L_{m}\right)=L_{1} \otimes\left(L_{2} \otimes L_{3} \otimes \cdots \otimes L_{m}\right)
\end{aligned}
$$

Equivalently, for arbitrary $\alpha \in \mathcal{T}(\mathcal{L})$,

$$
\begin{aligned}
& \vec{d}(\alpha)=\left(\operatorname{id}_{\mathcal{T}(\mathcal{L})} \otimes \Phi\right)\left(\Delta(\alpha)-\alpha \otimes 1_{\mathcal{T}(\mathcal{L})}\right) \\
& \overleftarrow{d}(\alpha)=\left(\Phi \otimes \operatorname{id}_{\mathcal{T}(\mathcal{L})}\right)\left(\Delta(\alpha)-1_{\mathcal{T}(\mathcal{L})} \otimes \alpha\right)
\end{aligned}
$$

where the enabling map $\Phi$ is the algebra homomorphism to $\mathcal{L}$ from the maximal ideal in $\mathcal{T}(\mathcal{L})$, consisting of tensors with vanishing homogeneous component of rank 0 , given by $\left(0, \alpha_{1}, \alpha_{2}, \ldots\right) \mapsto \alpha_{1} . \vec{d}$ and $\overleftarrow{d}$ satisfy the Leibniz-Itô formulas

$$
\begin{aligned}
& \vec{d}(\alpha \beta)=\vec{d}(\alpha) \beta+\alpha \vec{d}(\beta)+\vec{d}(\alpha) \vec{d}(\beta), \\
& \overleftarrow{d}(\alpha \beta)=\overleftarrow{d}(\alpha) \beta+\alpha \overleftarrow{d}(\beta)+\overleftarrow{d}(\alpha) \overleftarrow{d}(\beta)
\end{aligned}
$$

in which $\mathcal{T}(\mathcal{L}) \otimes \mathcal{L}$ and $\mathcal{L} \otimes \mathcal{T}(\mathcal{L})$ are regarded as $\mathcal{T}(\mathcal{L})$-bimodules using the natural tensorial extensions of the multiplicative biaction of $\mathcal{T}(\mathcal{L})$ on itself, as well as associative algebras with the tensor product multiplication.

Below we shall use the fact that if either $\vec{d}(\alpha)=0$ or $\overleftarrow{d}(\alpha)=0$ and also $\varepsilon(\alpha)=0$ then $\alpha=0$.

By comparing actions on homogeneous product tensors, the coproduct map $\Delta$, from $\mathcal{T}(\mathcal{L})$ to

$$
\mathcal{T}(\mathcal{L}) \otimes \mathcal{T}(\mathcal{L})=\bigoplus_{n=0}^{\infty}\left(\mathcal{T}(\mathcal{L}) \otimes\left(\otimes^{n} \mathcal{L}\right)\right)=\bigoplus_{n=0}^{\infty}\left(\left(\otimes^{n} \mathcal{L}\right) \otimes \mathcal{T}(\mathcal{L})\right),
$$

is seen to be given by the Taylor expansions

$$
\Delta=\bigoplus_{n=0}^{\infty} \vec{d}^{(n)}=\bigoplus_{n=0}^{\infty} \overleftarrow{d}^{(n)}
$$

where the iterates $\vec{d}^{(n)}$ and $\overleftarrow{d}^{(n)}$ are the maps from $\mathcal{T}(\mathcal{L})$ to $\mathcal{T}(\mathcal{L}) \otimes\left(\otimes^{n} \mathcal{L}\right)$ and $\left(\otimes^{n} \mathcal{L}\right) \otimes \mathcal{T}(\mathcal{L})$ respectively defined by $\vec{d}^{(0)}=\overleftarrow{d}^{(0)}=\operatorname{id}_{\mathcal{T}(\mathcal{L})}, \vec{d}^{(1)}=\vec{d}, \overleftarrow{d}^{(1)}=\overleftarrow{d}$ and for $n>1$

$$
\vec{d}^{(n)}=\left(\vec{d} \otimes \mathrm{id}_{\otimes^{n-1} \mathcal{L}}\right) \vec{d}^{(n-1)}, \quad \overleftarrow{d}^{(n)}=\left(\mathrm{id}_{\otimes^{n-1} \mathcal{L}} \otimes \overleftarrow{d}\right) \overleftarrow{d}^{(n-1)}
$$

We obtain the Taylor-Maclaurin expansions

$$
\operatorname{id}_{\mathcal{T}(\mathcal{L})}=\left(\varepsilon \otimes \operatorname{id}_{\mathcal{T}(\mathcal{L})}\right) \bigoplus_{n=0}^{\infty} \vec{d}^{(n)}=\left(\operatorname{id}_{\mathcal{T}(\mathcal{L})} \otimes \varepsilon\right) \bigoplus_{n=0}^{\infty} \overleftarrow{d}^{(n)}
$$

using the counital properties

$$
\left(\varepsilon \otimes \operatorname{id}_{\mathcal{T}(\mathcal{L})}\right) \Delta=\left(\operatorname{id}_{\mathcal{T}(\mathcal{L})} \otimes \varepsilon\right) \Delta=\mathrm{id}_{\mathcal{T}(\mathcal{L})} .
$$

Because of (7) and (8), the restrictions of $\vec{d}$ and $\overleftarrow{d}$ to the symmetric subalgebra $\mathcal{S}(\mathcal{L})$ map $\mathcal{S}(\mathcal{L})$ to $\mathcal{S}(\mathcal{L}) \otimes \mathcal{L}$ and $\mathcal{L} \otimes S(\mathcal{L})$ respectively. They are related by

$$
\vec{d}\left\lceil\mathcal{S}(\mathcal{L})=\tau_{(2,1)} \overleftarrow{d} \uparrow_{\mathcal{S}(\mathcal{L})}\right.
$$

Thus in calculus in the enveloping algebra $\mathcal{U}$ it is unnecessary to introduce both right and left differential maps [4]. 


\section{Simple product integrals}

For $L \in \mathcal{L}$ the right "initial value problem"

$$
\vec{d}(X)=X \otimes L, \quad \varepsilon(X)=1,
$$

or its left equivalent, has a solution in $\mathcal{T}(\mathcal{L})$ only in the trivial case $L=0$. To get something nontrivial we must consider formal power series.

Given an associative algebra $\mathcal{A}$, the space $\mathcal{A} \llbracket h \rrbracket$ of formal power series with coefficients in $\mathcal{A}$ becomes an associative algebra using the convolution product

$$
\sum_{N=0}^{\infty} h^{N} A_{N} \sum_{N=0}^{\infty} h^{N} B_{N}=\sum_{N=0}^{\infty} h^{N} \sum_{j=1}^{N} A_{N-j} B_{j}
$$

which is unital if $\mathcal{A}$ is unital. Below we shall use the well known fact that $A[h] \in h \mathcal{A} \llbracket h \rrbracket$ is a formal power series with vanishing zero order term then $A[h]$ has a unique two sided quasiinverse of the same form, that is an element $A^{\prime}[h] \in h \mathcal{A} \llbracket h \rrbracket$ such that

$$
A[h]+A^{\prime}[h]+A[h] A^{\prime}[h]=A^{\prime}[h]+A[h]+A^{\prime}[h] A[h]=0 .
$$

(For a proof, see [6] for example.)

We regard $\mathcal{T}(\mathcal{L}) \llbracket h \rrbracket$ as an associative algebra with the convolution product derived from the Itô shuffle product. We extend the coproduct and counit maps of $\mathcal{T}(\mathcal{L})$ to $\mathcal{T}(\mathcal{L}) \llbracket h \rrbracket$ by action on coefficents, retaining the same notations $\Delta$ and $\varepsilon$ for the extended maps. Similarly we extend the differential maps $\vec{d}$ and $\overleftarrow{d}$ to $\mathcal{T}(\mathcal{L}) \llbracket h \rrbracket$ by action on coefficients; then the Leibniz-Itô formulas continue to hold,

$$
\begin{aligned}
& \vec{d}(\alpha[h] \beta[h])=\vec{d}(\alpha[h]) \beta[h]+\alpha[h] \vec{d}(\beta[h])+\vec{d}(\alpha[h]) \vec{d}(\beta[h]), \\
& \overleftarrow{d}(\alpha[h] \beta[h])=\overleftarrow{d}(\alpha[h]) \beta[h]+\alpha[h] \overleftarrow{d}(\beta[h])+\overleftarrow{d}(\alpha[h]) \overleftarrow{d}(\beta[h])
\end{aligned}
$$

where the actions of $\mathcal{T}(\mathcal{L}) \llbracket h \rrbracket$ on $(\mathcal{T}(\mathcal{L}) \otimes \mathcal{L}) \llbracket h \rrbracket$ and $(\mathcal{L} \otimes \mathcal{T}(\mathcal{L})) \llbracket h \rrbracket$ are defined by combining convolution with the previously defined actions of $\mathcal{T}(\mathcal{L})$ on $(\mathcal{T}(\mathcal{L}) \otimes \mathcal{L})$ and $(\mathcal{L} \otimes \mathcal{T}(\mathcal{L}))$.

In the theorem which follows tensor products of formal power series are formal power series with coefficients formed by tensor product convolution, for example for $X[h]=\sum_{N=0}^{\infty} h^{N} X_{N}, X[h] \otimes X[h]=\sum_{N=0}^{\infty} h^{N} X_{N-j} \otimes$ $X_{j}$. For an element $L[h] \in h \mathcal{L} \llbracket h \rrbracket$, that is, a formal power series with coefficients in $\mathcal{L}$ whose zero order term vanishes, evidently $\otimes^{n} L[h] \in h^{n}\left(\otimes^{n} \mathcal{L}\right) \llbracket h \rrbracket$, so that $\bigoplus_{n=0}^{\infty} \otimes^{n} L[h]$ is a well defined element of $\mathcal{T}(\mathcal{L}) \llbracket h \rrbracket$.

Theorem 1. For an element $X[h]$ of $\mathcal{T}(\mathcal{L}) \llbracket h \rrbracket$ of the form $1_{\mathcal{T}(\mathcal{L})}+\mathrm{o}(h)$, the following conditions are equivalent;

(a): $\quad \Delta X[h]=X[h] \otimes X[h], \quad \varepsilon X[h]=1$.

(b): $\quad X[h]=\bigoplus_{n=0}^{\infty} \otimes^{n} L[h]$ for some $L[h] \in h \mathcal{L} \llbracket h \rrbracket$.

Proof. If (a) holds then, iterating, for $n \geqslant 1, \Delta^{(n)} X[h]=\otimes^{n} X[h]$, and hence, using (6), for $n>0,(X[h])_{n}=$ $\otimes^{n} L[h]$ where $L[h]=(X[h])_{1} \in h \mathcal{L} \llbracket h \rrbracket$. Also $(X[h])_{0}=\varepsilon X[h]=1$. Hence (b) holds. Conversely if (b) holds then

$$
\Delta X[h]=\bigoplus_{n=0}^{\infty} \sum_{j=0}^{n}\left(\otimes^{n-j} L[h]\right) \otimes\left(\otimes^{j} L[h]\right)=\left(\bigoplus_{n=0}^{\infty} \otimes^{n} L[h]\right) \otimes\left(\bigoplus_{n=0}^{\infty} \otimes^{n} L[h]\right)=X[h] \otimes X[h] ;
$$

also $\varepsilon X[h]=\otimes^{0}(L[h])=1$. Hence (a) holds. 
For $X[h]$ satisfying the conditions of Theorem 1 we write $X[h]=\prod(1+\mathrm{d} L[h])$ and call it the simple product integral generated by $L[h]$.

Another useful description of the simple product integral $X[h]=\prod(1+\mathrm{d} L[h])$ is that it is the unique solution of the differential equation

$$
\vec{d} X[h]=X[h] \otimes L[h], \quad \varepsilon X[h]=1 .
$$

Indeed it is clear from the expansion (b) of the theorem and from the second part of condition (a) that $X[h]=\prod(1+$ $\mathrm{d} L[h])$ satisfies (14). But if $X^{\prime}[h]$ is a second solution then the difference $Z[h]=X[h]-X^{\prime}[h]$ satisfies $\vec{d} Z[h]=0$, $\varepsilon Z[h]=0$. Hence $Z[h]=0$ and we have uniqueness. We use this characterisation to prove the following.

Proposition 2. $\prod(1+\mathrm{d} L[h])$ is invertible, with inverse $\prod\left(1+\mathrm{d} L^{\prime}[h]\right)$ where $L^{\prime}[h]$ is the quasiinverse of $L[h] \in$ $h \mathcal{L} \llbracket h \rrbracket$.

Proof. Let us prove that, for arbitrary $L[h], K[h] \in h \mathcal{L} \llbracket h \rrbracket$,

$$
\prod(1+\mathrm{d} L[h]) \prod(1+K[h])=\prod(1+d(L[h]+K[h]+L[h] K[h]))
$$

from which the proposition follows by taking $L[h]$ and $K[h]$ to be mutual quasiinverses and using the fact that when $L[h]=0, \Pi(1+\mathrm{d} L[h])=1_{\mathcal{T}(\mathcal{L})}$. Using the differential characterisation of product integrals and the Leibniz-Itô formula (12), we have, for $X[h]=\prod(1+\mathrm{d} L[h]), Y[h]=\prod(1+\mathrm{d} K[h])$,

$$
\begin{aligned}
\vec{d}(X[h] Y[h]) & =(X[h] \otimes L[h]) Y[h]+X[h](Y[h] \otimes L[h])+(X[h] \otimes L[h])(Y[h] \otimes L[h]) \\
& =X[h] Y[h] \otimes(L[h]+K[h]+L[h] K[h]) .
\end{aligned}
$$

Also by multiplicativity of $\varepsilon$,

$$
\varepsilon(X[h] Y[h])=\varepsilon(X[h]) \varepsilon(Y[h])=1 .
$$

Hence $Z[h]=X[h] Y[h]$ is the unique solution of

$$
\vec{d} Z[h]=Z[h] \otimes(L[h]+K[h]+L[h] K[h]), \quad \varepsilon Z[h]=1,
$$

that is $\prod(1+d(L[h]+K[h]+L[h] K[h]))$ as claimed.

Corollary 3. For mutually quasiinverse $L[h], L^{\prime}[h] \in h \mathcal{L} \llbracket h \rrbracket$ the map

$$
J[h]: \mathcal{T}(\mathcal{L}) \longrightarrow \mathcal{T}(\mathcal{L}) \llbracket h \rrbracket ; \quad \alpha \longmapsto \prod(1+d L[h]) \alpha \prod\left(1+L^{\prime}[h]\right)
$$

is unital and multiplicative.

Note that $\prod(1+\mathrm{d} L[h]) \in \mathcal{S}(\mathcal{L}) \llbracket h \rrbracket$ and that $J[h]$ maps $\mathcal{S}(\mathcal{L})$ to $\mathcal{S}(\mathcal{L}) \llbracket h \rrbracket$.

\section{Simple flows}

We say that a map $J[h]: \mathcal{T}(\mathcal{L})=\bigoplus_{n=0}^{\infty}\left(\otimes^{n} \mathcal{L}\right) \longrightarrow \mathcal{T}(\mathcal{L}) \llbracket h \rrbracket=\left(\bigoplus_{n=0}^{\infty}\left(\otimes^{n} \mathcal{L}\right)\right) \llbracket h \rrbracket$ is graded if it maps each homogeneous component space $\otimes^{n} \mathcal{L}$ to $\left(\otimes^{n} \mathcal{L}\right) \llbracket h \rrbracket$.

Theorem 4. For a linear map

$$
J[h]: \mathcal{T}(\mathcal{L}) \longrightarrow \mathcal{T}(\mathcal{L}) \llbracket h \rrbracket=\left(\bigoplus_{n=0}^{\infty}\left(\otimes^{n} \mathcal{L}\right)\right) \llbracket h \rrbracket
$$

of the form $\operatorname{id}_{\mathcal{T}(\mathcal{L})}+\mathrm{o}(h)$, the following conditions are equivalent. 
(a) $J[h]$ is graded and $\Delta J[h]=(J[h] \otimes J[h]) \Delta, \varepsilon J[h]=\varepsilon$;

(b) For $n=0,1,2, \ldots$ and $L_{1}, L_{2}, \ldots, L_{n} \in \mathcal{L},[h]\left(\otimes^{n} L_{j}\right)=\otimes^{n}\left(\left(\mathrm{id}_{\mathcal{L}}+j[h]\right)\left(L_{j}\right)\right)$ for some linear map $j[h]: \mathcal{L} \rightarrow h \mathcal{L} \llbracket h \rrbracket$.

Proof. Suppose (a) holds. Define $j[h]$ by $J[h](0, L, 0,0, \ldots)=\left(0,\left(\operatorname{id}_{\mathcal{L}}+j[h]\right) L, 0,0, \ldots\right)$. Then $j[h]$ inherits linearity from $J[h]$ and, since $J[h]=\mathrm{id}_{\mathcal{T}(\mathcal{L})}+\mathrm{o}(h), j[h]$ maps $\mathcal{L}$ to $h \mathcal{L} \llbracket h \rrbracket$. By $(6)$, for $n>0$ and $L_{1}, L_{2}, \ldots, L_{n} \in$ $\mathcal{L}$, since $J[h]$ is graded,

$$
\begin{aligned}
& J[h]\left(\otimes^{n} L_{j}\right)=\left(J[h]\left(\otimes^{n} L_{j}\right)\right)_{n}=\left(\Delta^{(n)} J[h]\left(\otimes^{n} L_{j}\right)\right)_{(1,1, \ldots, 1)}^{\left({ }_{n}\right)}\left(\left(\otimes^{n} J[h]\right)\left(\Delta^{(n)}\left(\otimes^{n} L_{j}\right)\right)\right)_{(1,1, \ldots, 1} \quad(n) \\
& =\left(\otimes^{n} J[h]\right)\left(\Delta^{(n)}\left(\otimes^{n} L_{j}\right)\right)_{(1,1, \ldots, 1)}{ }_{(n)}=\left(\otimes^{n} J[h]\right)\left(\otimes^{n} L_{j}\right)=\otimes^{n}\left(\left(\operatorname{id}_{\mathcal{L}}+j[h]\right)\left(L_{j}\right)\right) .
\end{aligned}
$$

Also, again because $J[h]$ is graded,

$$
J[h]\left(1_{\mathcal{T}(\mathcal{L})}\right)=\left(\varepsilon\left(J[h]\left(1_{\mathcal{T}(\mathcal{L})}\right)\right), 0,0, \ldots\right)=\left(\varepsilon\left(1_{\mathcal{T}(\mathcal{L})}\right), 0,0, \ldots\right)=1_{\mathcal{T}(\mathcal{L})} .
$$

Hence (b) holds.

Conversely suppose that (b) holds. Then for each homogeneous product tensor $L_{1} \otimes L_{2} \otimes \cdots \otimes L_{m}=\otimes^{m} L_{j}$

$$
J[h]\left(\otimes^{m} L_{j}\right)=\otimes^{m}\left(\left(\operatorname{id}_{\mathcal{L}}+j[h]\right)\left(L_{j}\right)\right)
$$

and so since $J[h]$ is unital,

$$
\begin{aligned}
\Delta J[h]\left(\otimes^{m} L_{j}\right) & =\Delta\left(\otimes^{m}\left(\left(\operatorname{id}_{\mathcal{L}}+j[h]\right)\left(L_{j}\right)\right)\right)=\sum_{k=0}^{m} \otimes^{k}\left(\left(\operatorname{id}_{\mathcal{L}}+j[h]\right)\left(L_{j}\right)\right) \otimes\left(\otimes_{l=k+1}^{m}\left(\left(\operatorname{id}_{\mathcal{L}}+j[h]\right)\left(L_{j}\right)\right)\right) \\
& =(J[h] \otimes J[h]) \Delta\left(L_{1} \otimes L_{2} \otimes \cdots \otimes L_{m}\right) .
\end{aligned}
$$

Also $\varepsilon J[h]=\varepsilon$. So (a) holds as required.

For $J[h]$ satisfying the conditions of Theorem 4 we write $J[h]=\prod(\mathrm{id}+\mathrm{d} j[h])$ and call it the simple flow generated by $j[h]$.

Corresponding to the differential description of simple product integrals we have the following characterisation of the flow generated by $j$; it is the unique solution of the differential equation

$$
\vec{d} J[h]=\left(J[h] \otimes\left(\operatorname{id}_{\mathcal{L}}+j[h]\right)\right) \vec{d}, \quad \varepsilon J[h]=\varepsilon .
$$

Indeed it is clear from the expansion (b) of the theorem and from the second part of condition (a) that $J[h]=$ $\prod\left(\operatorname{id}_{\mathcal{L}}+\mathrm{d} j[h]\right)$ satisfies (17). But if $J^{\prime}[h]$ is a second solution then the difference $K[h]=J[h]-J^{\prime}[h]$ satisfies

$$
\vec{d} K[h]=\left(K[h] \otimes\left(\operatorname{id}_{\mathcal{L}}+j[h]\right)\right) \vec{d}, \quad \varepsilon K[h]=0 .
$$

Using the Taylor-Maclaurin expansion, we have

$$
\begin{aligned}
K[h] & =\operatorname{id}_{\mathcal{T}(\mathcal{L})} K[h]=\left(\varepsilon \otimes \operatorname{id}_{\mathcal{T}(\mathcal{L})}\right) \bigoplus_{n=0}^{\infty} \vec{d}^{(n)} K[h] \\
& =\left(\varepsilon \otimes \operatorname{id}_{\mathcal{T}(\mathcal{L})}\right)\left(K[h] \otimes \bigoplus_{n=0}^{\infty} \otimes^{n}\left(\operatorname{id}_{\mathcal{L}}+j[h]\right) \vec{d}^{(n)}\right) \\
& =\varepsilon K[h] \otimes \bigoplus_{n=0}^{\infty} \otimes^{n}\left(\operatorname{id}_{\mathcal{L}}+j[h]\right) \vec{d}^{(n)}=0
\end{aligned}
$$

Hence we have uniqueness. 
(16) defines a flow of which the generator is given by

$$
j[h](K)=L[h] K+K L^{\prime}[h]+L[h] K L^{\prime}[h] .
$$

We call such a flow inner. Provided that $L[h]$ and $L^{\prime}[h]$ are mutually quasiinverse, the flow (16) is multiplicative. It may be verified directly that the generator $j[h]$ given by (19) is quasimultiplicative, that is, for arbitrary $K_{1}, K_{2} \in \mathcal{L}$,

$$
j[h]\left(K_{1} K_{2}\right)=j[h]\left(K_{1}\right) K_{2}+K_{1} j[h]\left(K_{2}\right)+j[h]\left(K_{1}\right) j[h]\left(K_{2}\right) .
$$

More generally we have the following.

Theorem 5. For the simple flow $J[h]$ generated by $j[h]$ to be multiplicative it is necessary and sufficient that $j[h]$ be quasimultiplicative from $\mathcal{L}$ to $\mathcal{L} \llbracket h \rrbracket$.

Proof. Suppose that $J[h]$ is multiplicative. Comparing the homogeneous components of rank 1 , of

$$
J[h](0, L, 0,0, \ldots) J[h](0, K, 0,0, \ldots)=J[h]((0, L, 0,0, \ldots)(0, K, 0,0, \ldots))
$$

using (2) we find that, for arbitrary $L, K \in \mathcal{L},\left(\operatorname{id}_{\mathcal{L}}+j[h]\right)(L K)=\left(\operatorname{id}_{\mathcal{L}}+j[h]\right)(L)\left(\operatorname{id}_{\mathcal{L}}+j[h]\right)(K)$, that is $j[h]$ is quasimultiplicative.

Suppose now that $j[h]$ is quasimultiplicative. Then $\sigma[h]=\operatorname{id}_{\mathcal{L}}+j[h]$ is multiplicative and so, using (2), we find that, for arbitrary $L_{1}, L_{2}, \ldots, L_{m+n} \in \mathcal{L}$,

$$
\begin{aligned}
J & {[h]\left(L_{1} \otimes L_{2} \otimes \cdots \otimes L_{m}\right) J[h]\left(L_{m+1} \otimes L_{m+2} \otimes \cdots \otimes L_{m+n}\right) } \\
& =\left(\sigma[h] L_{1} \otimes \sigma[h] L_{2} \otimes \cdots \otimes \sigma[h] L_{m}\right)\left(\sigma[h] L_{m+1} \otimes \sigma[h] L_{m+2} \otimes \cdots \otimes \sigma[h] L_{m}\right) \\
& =\sum_{P \in \mathcal{P}_{m, n}}\left(\sigma[h] L_{P_{1}} \otimes \sigma[h] L_{P_{2}} \otimes \cdots \otimes \sigma[h] L_{P_{k}}\right) \\
& =J[h] \sum_{P \in \mathcal{P}_{m, n}}\left(L_{P_{1}} \otimes L_{P_{2}} \otimes \cdots \otimes L_{P_{k}}\right) \\
& =J[h]\left(\left(L_{1} \otimes L_{2} \otimes \cdots \otimes L_{m}\right)\left(L_{m+1} \otimes L_{m+2} \otimes \cdots \otimes L_{m+n}\right)\right) .
\end{aligned}
$$

Hence $J[h]$ is multiplicative.

\section{Double product integrals}

To understand the definition of double product integrals which follows, let us first consider a discrete double product of elements $x_{j ; k}$ of elements of a unital associative algebra indexed by a Cartesian product of ordered sets, which are weakly commuting in the sense that $x_{j, k}$ commutes with $x_{j^{\prime} k^{\prime}}$ whenever both $j \neq j^{\prime}$ and $k \neq k^{\prime}$. Then [9] we have equality between the iterated ordered simple products

$$
\overleftarrow{\prod_{k}}\left\{\prod_{j} x_{j ; k}\right\}=\vec{\prod}\left\{\overleftarrow{\left.\prod_{j} x_{j ; k}\right\}}\right.
$$

for example $x_{1 ; 2} x_{2 ; 2} x_{1 ; 1} x_{1 ; 2}=x_{1 ; 2} x_{1 ; 1} x_{2 ; 2} x_{1 ; 2}$ because $x_{2 ; 2}$ commutes with $x_{1 ; 1}$. We denote the common value of this iterated product by $\prod_{j, k}^{\leftarrow} x_{j ; k}$.

Now let $\mathcal{M}_{m, n}$ be the set of $m \times n$ incidence matrices having the property that every row and every column contains at least one entry 1 . There are one-one correspondences between the elements $M$ of $\mathcal{M}_{m, n}$ and, on the one hand, the set of ordered $m$-tuples $\left(A_{1}, A_{2}, \ldots, A_{m}\right)$ of nonempty subsets whose union is $\mathbb{N}_{n}$, and, on the other hand, the set of ordered $n$-tuples $\left(B_{1}, B_{2}, \ldots, B_{n}\right)$ of nonempty subsets whose union is $\mathbb{N}_{m}$ given by

$$
M_{j, k}=1 \Longleftrightarrow k \in A_{j} \Longleftrightarrow j \in B_{k} .
$$


Now let $r[h] \in h(\mathcal{L} \otimes \mathcal{L}) \llbracket h \rrbracket$ and let $m, n \in \mathbb{N}$ and $M \in \mathcal{M}_{m, n}$. Then we can form the double product

$$
\prod_{(j, k) \in \mathbb{N}_{m} \times \mathbb{N}_{n}}^{\leftarrow}\left(r[h]^{M_{j, k}}\right)^{j ; k}=\overleftrightarrow{\prod_{j=1}^{m}}\left\{\prod_{k=1}^{\overleftarrow{n}}\left(r[h]^{M_{j, k}}\right)^{j ; k}\right\}=\overleftrightarrow{\prod_{k=1}^{n}}\left\{\prod_{j=1}^{\vec{m}}\left(r[h]^{M_{j, k}}\right)^{j ; k}\right\}
$$

in which, if $M_{j, k}=1,\left(r[h]^{M_{j, k}}\right)^{j ; k}$ is a copy of the formal power series $r[h]$ whose coefficients occupy the tensor product of the $j$ th copy of $\mathcal{L}$ in $\otimes^{m} \mathcal{L}$ with the $k$ th copy of $\mathcal{L}$ in $\otimes^{n} \mathcal{L}$, while, if $M_{j, k}=0,\left(r[h]^{M_{j, k}}\right)^{j ; k}$ is absent from the double product (or formally 1). Since the elements $x_{j, k}=\left(r[h]^{M_{j, k}}\right)^{j ; k}$ are formally weakly commuting and since every row and every column of $M$ contains a 1, this is a well defined element of $\left(\left(\otimes^{m} \mathcal{L}\right) \otimes\left(\otimes^{n} \mathcal{L}\right)\right) \llbracket h \rrbracket$. The backward-forward product $\overleftarrow{\prod}_{(j, k) \in \mathbb{N}_{m} \times \mathbb{N}_{n}}\left(r[h]^{M_{j, k}}\right)^{j ; k}$ is defined similarly.

Using the one-one correspondences (21) we have

$$
\begin{aligned}
\sum_{M \in \mathcal{M}_{m, n}} \prod_{(j, k) \in \mathbb{N}_{m} \times \mathbb{N}_{n}}^{\leftarrow}\left(r[h]^{M_{j, k}}\right)^{j ; k} & =\sum_{A_{1} \cup A_{2} \cup \ldots \cup A_{m}=\mathbb{N}_{n}} \prod_{j \in \mathbb{N}_{m}}^{\rightarrow} r[h]^{j ; A_{j}} \\
& =\sum_{B_{1} \cup B_{2} \cup \cdots \cup B_{n}=\mathbb{N}_{m}} \prod_{k \in \mathbb{N}_{n}} r[h]^{B_{k} ; k}
\end{aligned}
$$

where for $A_{j}=\left\{a_{1}>a_{2}>\cdots>a_{\left|A_{j}\right|}\right\}$ and $B_{k}=\left\{b_{1}<b_{2}<\cdots<b_{\left|B_{k}\right|}\right\}$

$$
r[h]^{j ; A_{j}}=r[h]^{j ; a_{1}} r[h]^{j ; a_{2}} \cdots r[h]^{j ; a_{\left|A_{j}\right|}}, \quad r[h]^{B_{k} ; k}=r[h]^{b_{1} ; k} r[h]^{b_{2} ; k} \cdots[h]^{b_{\left|B_{k}\right|} \mid k} .
$$

Now we can state our third characterisation theorem.

Theorem 6. For an element $X[h]$ of $(\mathcal{T}(\mathcal{L}) \otimes \mathcal{T}(\mathcal{L})) \llbracket h \rrbracket$ of the form $1_{\mathcal{T}(\mathcal{L}) \otimes \mathcal{T}(\mathcal{L})}+\mathrm{o}(h)$, the following conditions are equivalent;

$\begin{array}{ll}\text { (a): } & \left(\Delta \otimes \operatorname{id}_{\mathcal{T}(\mathcal{L})}\right) X[h]=X[h]^{1,3} X[h]^{2,3}, \quad\left(\varepsilon \otimes \operatorname{id}_{\mathcal{T}(\mathcal{L})}\right) X[h]=1, \\ & \left(\operatorname{id}_{\mathcal{T}(\mathcal{L})} \otimes \Delta\right) X[h]=X[h]^{1,3} X[h]^{1,2}, \quad\left(\operatorname{id}_{\mathcal{T}(\mathcal{L})} \otimes \varepsilon\right) X[h]=1 ;\end{array}$

(b): $\quad X[h]=1 \oplus \bigoplus_{m, n=1}^{\infty} \sum_{M \in \mathcal{M}_{m, n}} \prod_{(j, k) \in \mathbb{N}_{m} \times \mathbb{N}_{n}}^{\rightarrow}\left(r[h]^{M_{j, k}}\right)^{j ; k} \quad$ for some $r[h] \in h(\mathcal{L} \otimes \mathcal{L}) \llbracket h \rrbracket$.

Proof. If (a) holds then by the counital conditions the component of rank $(0 ; 0)$ of $X[h]$ is $1 \in \mathbb{C}=\mathbb{C} \otimes \mathbb{C}$, while those of ranks $(m ; 0)$ and $(0 ; n)$ for $m, n>0$ vanish. Also, by iteration of the coproduct conditions we obtain, for $m, n \geqslant 1$,

$$
\left(\Delta^{(m)} \otimes \Delta^{(n)}\right) X[h]=\prod_{(j, k) \in \mathbb{N}_{m} \times \mathbb{N}_{n}}^{\rightarrow \leftarrow} X[h]^{j ; k}
$$

where now the ordered discrete double product is an element of $\left(\left(\otimes^{m} \mathcal{T}(\mathcal{L})\right) \otimes\left(\otimes^{n} \mathcal{T}(\mathcal{L})\right)\right) \llbracket h \rrbracket$. Using $(6)$ we deduce that the component $X_{m ; n}[h]$ of joint rank $(m ; n)$ of $X[h]$ in $\left(\mathcal{T}(\mathcal{L}) \otimes \mathcal{T}(\mathcal{L}) \llbracket h \rrbracket=\bigoplus_{m, n=0}^{\infty}\left(\left(\otimes^{m} \mathcal{L}\right) \otimes\left(\otimes^{n} \mathcal{L}\right)\right) \llbracket h \rrbracket\right.$ is

$$
\left.\left(\left(\Delta^{(m)} \otimes \Delta^{(n)}\right) X[h]\right)_{(1,1, \ldots, 1) ;(1,1, \ldots, 1)}^{(m)} \cdot \underset{(j, k) \in \mathbb{N}_{m} \times \mathbb{N}_{n}}{\rightarrow \leftarrow} X[h]^{j ; k}\right)_{(1,1, \ldots, 1) ;(1,1, \ldots, 1)}^{(m)} .
$$


Only components of joint ranks $(0 ; 0),(1 ; 0),(0 ; 1)$ and $(1 ; 1)$ of $X[h]$ can contribute to this. The first of these components is 1 and the second and third vanish, as proved above; we denote the third by $r[h]$. Since $X[h]=$ $1_{\mathcal{T}(\mathcal{L}) \otimes \mathcal{T}(\mathcal{L})}+\mathrm{o}(h), r[h] \in h(\mathcal{L} \otimes \mathcal{L}) \llbracket h \rrbracket$ and we have

$$
X_{m ; n}[h]=\left(\prod_{(j, k) \in \mathbb{N}_{m} \times \mathbb{N}_{n}}^{\leftarrow}\left(1+r[h]^{j ; k}\right)\right)_{(1,1, \ldots, 1) ;(1,1, \ldots, 1)}^{(m)}=\sum_{M \in \mathcal{M}_{m, n}} \prod_{(j, k) \in \mathbb{N}_{m} \times \mathbb{N}_{n}}^{\leftarrow}\left(r[h]^{M_{j, k}}\right)^{j ; k}
$$

Conversely, assuming (b) holds, then, using (23)

$$
\begin{aligned}
& \left(\Delta \otimes \operatorname{id}_{\mathcal{T}(\mathcal{L})}\right) X[h] \\
& =\left(\Delta \otimes \operatorname{id}_{\mathcal{T}(\mathcal{L})}\right)\left(1 \oplus \bigoplus_{m, n=1}^{\infty} \sum_{M \in \mathcal{M}_{m, n}} \prod_{(j, k) \in \mathbb{N}_{m} \times \mathbb{N}_{n}}^{\leftarrow}\left(r[h]^{M_{j, k}}\right)^{j ; k}\right) \\
& =\left(\Delta \otimes \operatorname{id}_{\mathcal{T}(\mathcal{L})}\right)\left(1 \oplus \bigoplus_{m, n=1}^{\infty} \sum_{A_{1} \cup A_{2} \cup \cdots \cup A_{m}=\mathbb{N}_{n}} \vec{\prod}_{j \in \mathbb{N}_{m}} r[h]^{j ; A_{j}}\right) \\
& =1 \oplus \bigoplus_{m, n=1}^{\infty} \sum_{A_{1} \cup A_{2} \cup \cdots \cup A_{m}=\mathbb{N}_{n}} \sum_{k=0}^{m}\left(\prod_{j \in \mathbb{N}_{k}} r[h]^{j ; A_{j}}\right)\left(\prod_{j \in \mathbb{N}_{m}^{k}} r[h]^{j ; A_{j}}\right) \\
& =1 \oplus \bigoplus_{m, n=1}^{\infty} \sum_{k=0}^{m} \sum_{C \cup D=\mathbb{N}_{n}} \sum_{A_{1} \cup A_{2} \cup \cdots \cup A_{k}=C}\left(\overrightarrow{\prod_{j \in \mathbb{N}_{k}}} r[h]^{j ; A_{j}}\right)_{A_{k+1} \cup A_{k+2} \cup \ldots \cup A_{m}=D}\left(\overrightarrow{\prod_{j \in \mathbb{N}_{m}^{k}}} r[h]^{j ; A_{j}}\right) \\
& =1 \oplus \bigoplus_{m, n=1}^{\infty} \sum_{C \cup D=\mathbb{N}_{n}} \sum_{k=0}^{m}\left(X[h]_{k ;|C|}\right)^{\{1,2, \ldots, k\} ; C}\left(X[h]_{m-k ;|D|}\right)^{\{k+1, k+2, \ldots, m\} ; D} \\
& =X[h]^{1,3} X[h]^{2,3} \text {. }
\end{aligned}
$$

Also, since $(X[h])_{0 ; 0}=1$ and $(X[h])_{0 ; n}=0$ for $n>0$,

$\left(\varepsilon \otimes \operatorname{id}_{\mathcal{T}(\mathcal{L})}\right) X[h]=1$.

A similar argument starting with the alternative expression (24) for the double product shows that

$$
\left(\operatorname{id}_{\mathcal{T}(\mathcal{L})} \otimes \Delta\right) X[h]=X[h]^{1,3} X[h]^{1,2}, \quad\left(\operatorname{id}_{\mathcal{T}(\mathcal{L})} \otimes \varepsilon\right) X[h]=1 .
$$

Hence (a) holds as required.

For $X[h]$ satisfying the conditions of Theorem 6 we write $X[h]=\vec{\prod} \leftarrow(1+\mathrm{d} r[h])$ and call it the forwardbackward directed product integral generated by $r[h]$. The backward-forward directed product integral $\overleftrightarrow{\prod}(1+\mathrm{d} r[h])$ is defined analogously.

Except in the case when $\mathcal{L}$ is commutative, there is no analog for double product integrals of the rule (15) for multiplying simple product integrals used to prove Proposition 2. Despite this an analogous proposition holds.

Proposition 7. $\overleftrightarrow{\prod}(1+\mathrm{d} r[h])$ is invertible, with inverse $\overleftrightarrow{\prod}\left(1+\mathrm{d} r^{\prime}[h]\right)$ where $r^{\prime}[h]$ is the quasiinverse of $r[h] \in h(\mathcal{L} \otimes \mathcal{L}) \llbracket h \rrbracket$.

Proof. Let us prove that $\vec{\prod}(1+\mathrm{d} r[h]) \overleftrightarrow{\prod}\left(1+\mathrm{d} r^{\prime}[h]\right)=1_{\mathcal{T}(\mathcal{L}) \otimes \mathcal{T}(\mathcal{L})}$; the proof that $\overleftrightarrow{\prod}\left(1+\mathrm{d} r^{\prime}[h]\right) \vec{\prod}$ $(1+\mathrm{d} r[h])=1_{\mathcal{T}(\mathcal{L}) \otimes \mathcal{T}(\mathcal{L})}$ is similar. We have 


$$
\begin{aligned}
(\vec{d} & \left.\otimes \operatorname{id}_{\mathcal{T}(\mathcal{L})}\right) \vec{\prod}(1+\mathrm{d} r[h]) \\
& =\left(\vec{d} \otimes \operatorname{id}_{\mathcal{T}(\mathcal{L})}\right)\left(1 \oplus \bigoplus_{m, n=1}^{\infty} \sum_{A_{1} \cup A_{2} \cup \cdots \cup A_{m}=\mathbb{N}_{n}} \prod_{j \in \mathbb{N}_{m}} r[h]^{j ; A_{j}}\right) \\
& =\bigoplus_{m, n=1}^{\infty} \sum_{A_{1} \cup A_{2} \cup \ldots \cup A_{m}=\mathbb{N}_{n}}\left(\prod_{j \in \mathbb{N}_{m-1}} r[h]^{j ; A_{j}}\right)^{1,3} r[h]^{m ; A_{m}} \\
& =\bigoplus_{m, n=1}^{\infty}\left(\sum_{A_{1} \cup A_{2} \cup \ldots \cup A_{m-1}=\mathbb{N}_{n-1}} \prod_{j \in \mathbb{N}_{m-1}} r[h]^{j ; A_{j}}\right)^{1,3}\left(\bigoplus_{n=1}^{\infty}\left(r[h]^{2 ; n} r[h]^{2 ; n-1} \ldots r[h]^{2 ; 1}\right)\right)^{2,3}
\end{aligned}
$$

using the multiplication rule (3). Thus

$$
\left(\vec{d} \otimes \operatorname{id}_{\mathcal{T}(\mathcal{L})}\right) \vec{\prod}(1+\mathrm{d} r[h])=\left(\vec{\prod}(1+\mathrm{d} r[h])\right)^{1,3} P[h]^{2,3}
$$

where $P[h]=\bigoplus_{n=1}^{\infty}\left\{\coprod_{k=1}^{n} r[h]^{1 ; k}\right\}$. A similar argument shows that

$$
\left(\vec{d} \otimes \operatorname{id}_{\mathcal{T}(\mathcal{L})}\right) \overleftrightarrow{\prod}\left(1+\mathrm{d} r^{\prime}[h]\right)=Q[h]^{2,3}\left(\overleftrightarrow{\prod}\left(1+\mathrm{d} r^{\prime}[h]\right)\right)
$$

where $Q[h]=\bigoplus_{n=1}^{\infty}\left\{\left[\vec{\prod}_{k=1}^{n} r^{\prime}[h]^{1 ; k}\right\}\right.$. Hence by the Leibniz-Itô formula, we have

$$
\begin{aligned}
(\vec{d} & \left.\otimes \operatorname{id}_{\mathcal{T}(\mathcal{L})}\right)\left\{\vec{\prod}(1+\mathrm{d} r[h]) \overleftrightarrow{\prod^{\prime}}\left(1+\mathrm{d} r^{\prime}[h]\right)\right\} \\
& =\left(\vec{\prod}(1+\mathrm{d} r[h])\right)^{1,3}(P[h]+Q[h]+P[h] Q[h])^{2,3}\left(\overleftarrow{\prod}\left(1+\mathrm{d} r^{\prime}[h]\right)\right)^{1,3}
\end{aligned}
$$

But from the definitions of $P[h]$ and $Q[h]$,

$$
\begin{aligned}
& \left(\operatorname{id}_{\mathcal{L}} \otimes \overleftarrow{d}\right) P[h]=\left(1^{3}+P[h]^{2,3}\right) r[h]^{1,2}, \\
& \left(\operatorname{id}_{\mathcal{L}} \otimes \overleftarrow{d}\right) Q[h]=r^{\prime}[h]^{1,2}\left(1^{3}+Q[h]^{2,3}\right)
\end{aligned}
$$

and hence, again using the Leibniz-Itô formula,

$$
\begin{aligned}
\left(\operatorname{id}_{\mathcal{L}} \otimes\right. & \overleftarrow{d})(P[h]+Q[h]+P[h] Q[h]) \\
= & \left(1_{\mathcal{T}(\mathcal{L})}^{3}+P[h]^{2,3}\right) r[h]^{1,2}+r^{\prime}[h]^{1,2}\left(1_{\mathcal{T}(\mathcal{L})}^{3}+Q[h]^{2,3}\right) \\
& +\left(1_{\mathcal{T}(\mathcal{L})}^{3}+P[h]^{2,3}\right) r[h]^{1,2} r^{\prime}[h]^{1,2}\left(1_{\mathcal{T}(\mathcal{L})}^{3}+Q[h]^{2,3}\right) \\
= & \left.1_{\mathcal{T}(\mathcal{L})}^{3} r[h]^{1,2}+r^{\prime}[h]^{1,2} 1_{\mathcal{T}(\mathcal{L})}^{3}+1_{\mathcal{T}(\mathcal{L})}^{3} r h\right]^{1,2} r^{\prime}[h]^{1,2} 1_{\mathcal{T}(\mathcal{L})}^{3} \\
& +P[h]^{2,3}\left(r[h]^{1,2}+r^{\prime}[h]^{1,2}+r[h]^{1,2} r^{\prime}[h]^{1,2}\right) \\
& +\left(r[h]^{1,2}+r^{\prime}[h]^{1,2}+r[h]^{1,2} r^{\prime}[h]^{1,2}\right) Q[h]^{2,3}+P[h]^{2,3}\left(r[h]^{1,2}+r^{\prime}[h]^{1,2}+r[h]^{1,2} r^{\prime}[h]^{1,2}\right) Q[h]^{2,3}
\end{aligned}
$$

Rearranging the first three terms as $\left(r[h]^{1,2}+r^{\prime}[h]^{1,2}+r[h]^{1,2} r^{\prime}[h]^{1,2}\right) 1_{\mathcal{T}(\mathcal{L})}^{3}$ and using the fact that $r[h]$ and $r^{\prime}[h]$ are mutually quasiinverse we obtain

$$
\left(\operatorname{id}_{\mathcal{L}} \otimes \overleftarrow{d}\right)(P[h]+Q[h]+P[h] Q[h])=0
$$

Also, by multiplicativity of $\varepsilon$ and from the definitions of $P[h]$ and $Q[h]$ 


$$
\begin{aligned}
& \quad\left(\operatorname{id}_{\mathcal{L}} \otimes \varepsilon\right)(P[h]+Q[h]+P[h] Q[h]) \\
& \quad=\left(\operatorname{id}_{\mathcal{L}} \otimes \varepsilon\right) P[h]+\left(\operatorname{id}_{\mathcal{L}} \otimes \varepsilon\right) Q[h]+\left(\operatorname{id}_{\mathcal{L}} \otimes \varepsilon\right) P[h]\left(\operatorname{id}_{\mathcal{L}} \otimes \varepsilon\right) Q[h]=0 .
\end{aligned}
$$

From (26) and (27) it follows that $P[h]+Q[h]+P[h] Q[h]=0$. Hence, from (25),

$$
\left(\vec{d} \otimes \operatorname{id}_{\mathcal{T}(\mathcal{L})}\right)\left\{\vec{\prod}(1+\mathrm{d} r[h]) \overleftrightarrow{\prod}\left(1+\mathrm{d} r^{\prime}[h]\right)\right\}=0
$$

But by multiplicativity of $\varepsilon$,

$$
\begin{aligned}
& \left(\varepsilon \otimes \operatorname{id}_{\mathcal{T}(\mathcal{L})}\left\{\vec{\prod}(1+\mathrm{d} r[h]) \overleftrightarrow{\prod}\left(1+\mathrm{d} r^{\prime}[h]\right)\right\}\right. \\
& =\left(\varepsilon \otimes \operatorname{id}_{\mathcal{T}(\mathcal{L})}\left\{\vec{\prod}(1+\mathrm{d} r[h])\right\}\left(\varepsilon \otimes \operatorname{id}_{\mathcal{T}(\mathcal{L})}\right)\left\{\overleftrightarrow{\prod}\left(1+\mathrm{d}^{\prime}[h]\right)\right\}=1_{\mathcal{T}(\mathcal{L})}\right.
\end{aligned}
$$

Hence $\vec{\prod}(1+\mathrm{d} r[h]) \overleftrightarrow{\prod}\left(1+\mathrm{d} r^{\prime}[h]\right)=1_{\mathcal{T}(\mathcal{L}) \otimes \mathcal{T}(\mathcal{L}) \text { as required }}$

Corollary 8. For mutually quasiinverse $r[h], r^{\prime}[h] \in h(\mathcal{L} \otimes \mathcal{L}) \llbracket h \rrbracket$ the map

$$
J[h]: \mathcal{T}(\mathcal{L}) \otimes \mathcal{T}(\mathcal{L}) \longrightarrow(\mathcal{T}(\mathcal{L}) \otimes \mathcal{T}(\mathcal{L})) \llbracket h \rrbracket ; \quad \xi \longmapsto \overleftrightarrow{\prod}(1+\mathrm{d} r[h]) \xi \overleftrightarrow{\prod}\left(1+\mathrm{d} r^{\prime}[h]\right)
$$

is multiplicative.

\section{The quantisation problem for Lie bialgebras}

Let us consider further the multiplicative "inner double flow" $J[h]$ defined by Corollary 8 . We use the notations

$$
R[h]=\overleftrightarrow{\prod^{\leftarrow}}(1+\mathrm{d} r[h]), \quad R^{\prime}[h]=\overleftrightarrow{\prod^{\prime}}\left(1+\mathrm{d} r^{\prime}[h]\right)=(R[h])^{-1}
$$

The map $\Delta[h]$ from $\mathcal{T}(\mathcal{L})$ to $(\mathcal{T}(\mathcal{L}) \otimes \mathcal{T}(\mathcal{L})) \llbracket h \rrbracket$ given by $\Delta[h]=J[h] \Delta$ is a composition of multiplicative maps, hence multiplicative. Let us investigate when $\Delta[h]$ satisfies the coassociativity condition

$$
\left(\Delta[h] \otimes \operatorname{id}_{\mathcal{T}(\mathcal{L})}\right) \Delta[h]=\left(\mathrm{id}_{\mathcal{T}(\mathcal{L})} \otimes \Delta[h]\right) \Delta[h]
$$

where as usual compositions of formal power series whose coefficients are maps are formed by convolution. Using the definition of $\Delta[h]$ and the multiplicativity of $\Delta$, for arbitrary $\xi \in \mathcal{T}(\mathcal{L}) \otimes \mathcal{T}(\mathcal{L})$,

$$
\begin{aligned}
\left(\Delta[h] \otimes \operatorname{id}_{\mathcal{T}(\mathcal{L})}\right) \Delta[h](\xi) & =R[h]^{1,2}\left(\left(\Delta \otimes \mathrm{id}_{\mathcal{T}(\mathcal{L})}\right) \Delta[h](\xi)\right) R^{\prime}[h]^{1,2} \\
& =R[h]^{1,2}\left(\left(\Delta \otimes \mathrm{id}_{\mathcal{T}(\mathcal{L})}\right)\left(R[h] \Delta(\xi) R^{\prime}[h]\right)\right) R^{\prime}[h]^{1,2} \\
& =R[h]^{1,2}\left(\left(\Delta \otimes \mathrm{id}_{\mathcal{T}(\mathcal{L})}\right)(R[h])\left(\left(\Delta \otimes \mathrm{id}_{\mathcal{T}(\mathcal{L})}\right) \Delta(\xi)\right)\left(\Delta \otimes \operatorname{id}_{\mathcal{T}(\mathcal{L})}\right)\left(R^{\prime}[h]\right)\right) R^{\prime}[h]^{1,2} \\
& =R[h]^{1,2} R[h]^{1,3} R[h]^{2,3}\left(\left(\Delta \otimes \operatorname{id}_{\mathcal{T}(\mathcal{L})}\right) \Delta(\xi)\right) R^{\prime}[h]^{2,3} R^{\prime}[h]^{1,3} R^{\prime}[h]^{1,2}
\end{aligned}
$$

where we use the characterisation (a) of Theorem 6 of the forward-backward double product integral $R[h]$ together with the analogous characterisation of the backward-forward double product integral $R^{\prime}[h]$.

A similar argument shows that

$$
\left(\operatorname{id}_{\mathcal{T}(\mathcal{L})} \otimes \Delta[h]\right) \Delta[h](\xi)=R[h]^{2,3} R[h]^{1,3} R[h]^{1,2}\left(\left(\operatorname{id}_{\mathcal{T}(\mathcal{L})} \otimes \Delta\right) \Delta(\xi)\right) R^{\prime}[h]^{1,2} R^{\prime}[h]^{1,3} R^{\prime}[h]^{2,3} .
$$

Since $\Delta$ is coassociative, $\left(\Delta \otimes \operatorname{id}_{\mathcal{T}(\mathcal{L})}\right) \Delta(\xi)=\left(\operatorname{id}_{\mathcal{T}(\mathcal{L})} \otimes \Delta\right) \Delta(\xi)$ and it follows that $\Delta[h]$ is coassociative if $R[h]$ satisfies the quantum Yang-Baxter equation

$$
R[h]^{1,2} R[h]^{1,3} R[h]^{2,3}=R[h]^{2,3} R[h]^{1,3} R[h]^{1,2}
$$


in $(\mathcal{T}(\mathcal{L}) \otimes \mathcal{T}(\mathcal{L}) \otimes \mathcal{T}(\mathcal{L})) \llbracket h \rrbracket$ (in which case its inverse $R^{\prime}[h]$ satisfies the same equation). In [8] it is shown that a necessary and sufficient condition for $R[h]=\vec{\prod}(1+\mathrm{d} r[h])$ to satisfy (29) is that

$$
\begin{aligned}
& r[h]^{1,2} r[h]^{1,3}+r[h]^{1,2} r[h]^{2,3}+r[h]^{1,3} r[h]^{2,3}+r[h]^{1,2} r[h]^{1,3} r[h]^{2,3} \\
& \quad=r[h]^{1,3} r[h]^{1,2}+r[h]^{2,3} r[h]^{1,2}+r[h]^{2,3} r[h]^{1,3}+r[h]^{2,3} r[h]^{1,3} r[h]^{1,2}
\end{aligned}
$$

as elements of $(\mathcal{L} \otimes \mathcal{L} \otimes \mathcal{L}) \llbracket h \rrbracket$. This equation also occurs in [2]. In a footnote to [9] it was claimed erroneously to be a necessary and sufficient condition for the symmetrised double product integral generated by $r[h]$ to satisfy the quantum Yang-Baxter equation. It can be verified directly that if $r[h]$ satisfies (30) then its quasiinverse $r^{\prime}[h]$ satisfies the same equation. The argument of [8] can be modified to show that this is necessary and sufficient for $R^{\prime}[h]=\overleftrightarrow{\prod}\left(1+\mathrm{d} r^{\prime}[h]\right)$ to satisfy the quantum Yang-Baxter equation.

The condition (30) can be analysed by comparing coefficients of powers of $h[9,2]$; for the lowest power $h^{2}$ it is found that the first order coefficient $r_{1}$ of $r[h]$ must satisfy the classical Yang-Baxter equation

$$
\left[r_{1}^{1,2} r_{1}^{1,3}\right]+\left[r_{1}^{1,2}, r_{1}^{2,3}\right]+\left[r_{1}^{1,3}, r_{1}^{2,3}\right]=0
$$

while higher powers give a hierarchy of inhomogeneous linear equations for the successive coefficients $r_{2}, r_{3}, \ldots$, namely

$$
\begin{aligned}
& {\left[r_{n}^{1,2} r_{1}^{1,3}\right]+\left[r_{n}^{1,2}, r_{1}^{2,3}\right]+\left[r_{n}^{1,3}, r_{1}^{2,3}\right]+\left[r_{1}^{1,2} r_{n}^{1,3}\right]+\left[r_{1}^{1,2}, r_{n}^{2,3}\right]+\left[r_{1}^{1,3}, r_{n}^{2,3}\right]} \\
& \quad=-\sum_{s, t>1, s+t=n+1}\left(\left[r_{s}^{1,2} r_{t}^{1,3}\right]+\left[r_{s}^{1,2}, r_{t}^{2,3}\right]+\left[r_{s}^{1,3}, r_{t}^{2,3}\right]\right)-\sum_{s+t+u=n+1}\left(r_{s}^{1,2} r_{t}^{1,3} r_{u}^{2,3}-r_{s}^{2,3} r_{t}^{1,3} r_{u}^{1,2}\right) .
\end{aligned}
$$

Assuming that solutions of this hierarchy can be found we have thus constructed a deformation coproduct $\Delta[h]$ in the algebra $\mathcal{A}$ of formal power series with coefficients in the Itô-Hopf algebra $\mathcal{T}(\mathcal{L})$ whose zero-order coefficients are elements of the symmetric sub-Hopf algebra $\mathcal{S}(\mathcal{L})$ which is isomorphic to the universal enveloping algebra of the commutator Lie algebra of $\dot{\mathcal{L}}$. The Lie bialgebra of which this is the quantisation is determined by the Lie cobracket for $\dot{\mathcal{L}}$

$$
\begin{aligned}
\delta(L) & =h^{-1}\left(\Delta[h](L)-\Delta[h]^{\mathrm{opp}}(L)\right)+\mathrm{o}(h) \\
& =h^{-1}\left(R[h] \Delta(L) R^{\prime}[h]-\tau_{(2,1)}\left(R[h] \Delta(L) R^{\prime}[h]\right)\right)+\mathrm{o}(h) \\
& =h^{-1}\left((1+r[h]) \Delta(L)(1-r[h])-\tau_{(2,1)}(1+r[h]) \Delta(L)(1-r[h])\right)+\mathrm{o}(h) \\
& =\left[r_{1}-\tau_{(2,1)} r_{1}, L^{1}+L^{2}\right]
\end{aligned}
$$

where $\Delta[h]^{\text {opp }}$ is the opposite coproduct to $\Delta[h]$ got by replacing $r_{1}$ by $\tau_{(2,1)} r_{1}$ where $\tau_{(2,1)}$ is the flip map from $\mathcal{L} \otimes \mathcal{L}$ to itself which exchanges the components of product tensors. Here we use the expansion $\vec{\prod}(1+\mathrm{d} r[h])=$ $1+r[h]+\mathrm{o}\left(h^{2}\right)$. Thus, modulo the existence of solutions to the hierarchy, we have a general method of quantisation of so called quasitriangular Lie bialgebras, for which the cobracket is of this kind with $r_{1}$ satisfying the classical Yang-Baxter equation. It is natural to speculate that a more general theory of not necessarily inner double flows may similarly quantise more general Lie bialgebras.

In fact it follows from an argument of Enriquez that, as conjectured in [9], the hierarchy always has solutions. But Enriquez's argument uses the general existence theorem for a quantisation of a Lie bialgebra [3]. Thus the method of quantisation by double product integrals does not yet provide an alternative to the method of Etingof and Kazhdan which is based on constructing an associator from the monodromy properties of the KnizhnikZamolodchikov system of holomorphic differential equations. It seems unsatisfactory that a purely algebraic result, the existence of a quantisation of a general Lie bialgebra, depends on such an analytic mechanism. 


\section{References}

[1] L. Accardi, A. Frigerio, J.T. Lewis, Quantum stochastic processes, Publ. RIMS Kyoto 18 (1982) 97-133.

[2] B. Enriquez, Quantisation of Lie bialgebras and shuffle algebras of Lie algebras, Selecta Math. (N.S.) 7 (2001) $321-407$.

[3] P. Etingof, D. Kazhdan, Quantization of Lie bialgebras, I, Selecta Math. (NS.) 2 (1966) 1-41.

[4] R.L. Hudson, Calculus in enveloping algebras, J. London Math. Soc. (2) 65 (2002) 361-380.

[5] R.L. Hudson, K.R. Parthasarathy, Quantum Itô's formula and stochastic evolutions, Commun. Math. Phys. 93 (1984) $301-323$.

[6] R.L. Hudson, K.R. Parthasarathy, S. Pulmannová, The method of formal power series in quantum stochastic calculus, IDAQP 3 (2000) $387-401$.

[7] R.L. Hudson, S. Pulmannová, Double product integrals and Enriquez quantisation of Lie bialgebras I: the quasitriangularity relations, J. Math. Phys. 45 (2004) 2090-2105.

[8] R.L. Hudson, S. Pulmannová, Double product integrals and Enriquez quantisation of Lie bialgebras II: the quantum Yang-Baxter equation, Lett. Math. Phys., submitted for publication.

[9] R.L. Hudson, S. Pulmannová, Symmetrized double quantum stochastic product integrals, J. Math. Phys. 41 (2000) $8249-8262$.

[10] P.A. Meyer, Quantum Probability for Probabilists, Lecture Notes in Math., vol. 1538, Springer, 1993.

[11] R.F. Streater, Current commutation relations, continuous tensor products and infinitely divisible group representations, Rend. Sc. Inst. Fisica E. Fermi XI (1969) 247-263. 